\title{
Correlative Ultrastructural Analysis of Functionally Modulated Synapses Using Automatic Tape-Collecting Ultramicrotome - SEM Array Tomography.
}

\author{
Naomi Kamasawa ${ }^{1}$, Ye Sun $^{2}$, Takayasu Mikuni ${ }^{2}$, Debbie Guerrero-Given ${ }^{1}$, Ryohei Yasuda ${ }^{2}$ \\ ${ }^{1}$ Electron Microscopy Core Facility, Max Planck Florida Institute for Neuroscience, Jupiter, USA \\ ${ }^{2}$ Neuronal Signal Transduction, Max Planck Florida Institute for Neuroscience, Jupiter, USA
}

To understand synaptic plasticity, correlative microscopic approaches using functional imaging by light microscopy combined with electron microscopy (EM) are necessary. Ultrastructure of synapses can be visualized only by EM, but re-finding the same synapses that were manipulated and imaged by light microscope in the EM is a very laborious and time-consuming task when performing the conventional serial ultrathin-sectioning and observation method with transmission EM. Recently, new imaging applications to obtain volume data sets from brain tissues were introduced using an automatic tapecollecting microtome (ATUM) [1] or a focused ion beam [2] with scanning electron microscopy (SEM). We used an ATUMtome (RMC/Boeckeler) and automatic array tomography software, Atlas AT5 (Zeiss) and established a workflow to investigate ultrastructural changes of synapses. We first performed glutamate uncaging by 2-photon laser microscope to induce/modify spines, and obtained the whole cell image with a confocal microscope. Then the tissues are prepared with conventional pre-embedding immuno-EM using nanogold labeling for EM visualization as explained below.

2-photon glutamate uncaging and confocal laser scanning microscopy (CLSM) imaging: We uncaged MNI-glutamate with $4 \mathrm{~mW}(720 \mathrm{~nm}, 2$ photon), $2 \mathrm{~Hz}$ X 40 stimulation on smooth dendrites of pyramidal neurons expressing EGFP in mouse hippocampal organotypic slice cultures. After the new spine formation visually confirmed, tissue slices were fixed in a mixture of $4 \%$ paraformaldehyde and 0.5-1\% glutaraldehyde. CLSM Z-stack images were captured with a lower magnification to overview the whole tissue slice containing GFP fluorescent cells. Once the target neuron was identified, it was imaged to visualize its dendritic arbor and to define the position of the newly developed spines.

Immuno-staining and resin embedding: Slices were cryo-protected with sucrose, permeabilized by liquid nitrogen, blocked and immunostained with anti-GFP antibody followed by nanogold conjugated secondary antibody. Slices were then silver enhanced, post-fixed with $\mathrm{OsO}_{4}$, en-block stained with uranyl acetate, dehydrated with a series of ethanol and acetone, and embedded in Durcupan resin.

ATUM and SEM imaging with Atlas AT5/ Merlin VP Compact (Zeiss): The area containing the target cell was trimmed based on the CLSM image to a size of $1 \times 1.5 \mathrm{~mm}$. The sample was sectioned at 60-70 nm thickness with an ATUMtome and collected on Kapton tape automatically, then the kapton tape was placed on silicon wafers. The overview image of the Si wafer was captured using a digital camera, the image was loaded into the Atlas AT5 and aligned to the physical location of the wafer in the SEM using mesh grids as fiducials on the wafer. Low-resolution SE2 images for all sections were first captured with a $2 \mu \mathrm{m} /$ pixel xy-resolution protocol $\left(4 \mathrm{~mm}^{2}\right.$ imaging area) and overlaid on the overview image. An approximate area of the target cell was demarcated on the serial sections, and montage images were captured with a $30 \mathrm{~nm}$ pixel resolution protocol using BSE. After finding the target cell, a rough 3D reconstruction was performed to see the orientation of the target dendrite and spine. The high-resolution images of the target spine were captured with a 4-7 $\mathrm{nm}$ pixel resolution protocol using an InlensDuo detector in the BSE mode. 
Summary: This workflow allowed analyzing morphologies of synaptic plasticity from 2-photon microscope to EM. By using a CLSM fluorescent Z-stack image as a bridge, immunogold labeled profiles that were functionally modulated and imaged by 2-photon microscope were relatively easily relocated without adding a fiducial- marking within the tissue. Three different detectors were used to fit to the best performance of imaging time and quality at each step of the workflow. It is still timeconsuming, but could be handled routinely and useful to obtain quantitative data sets.

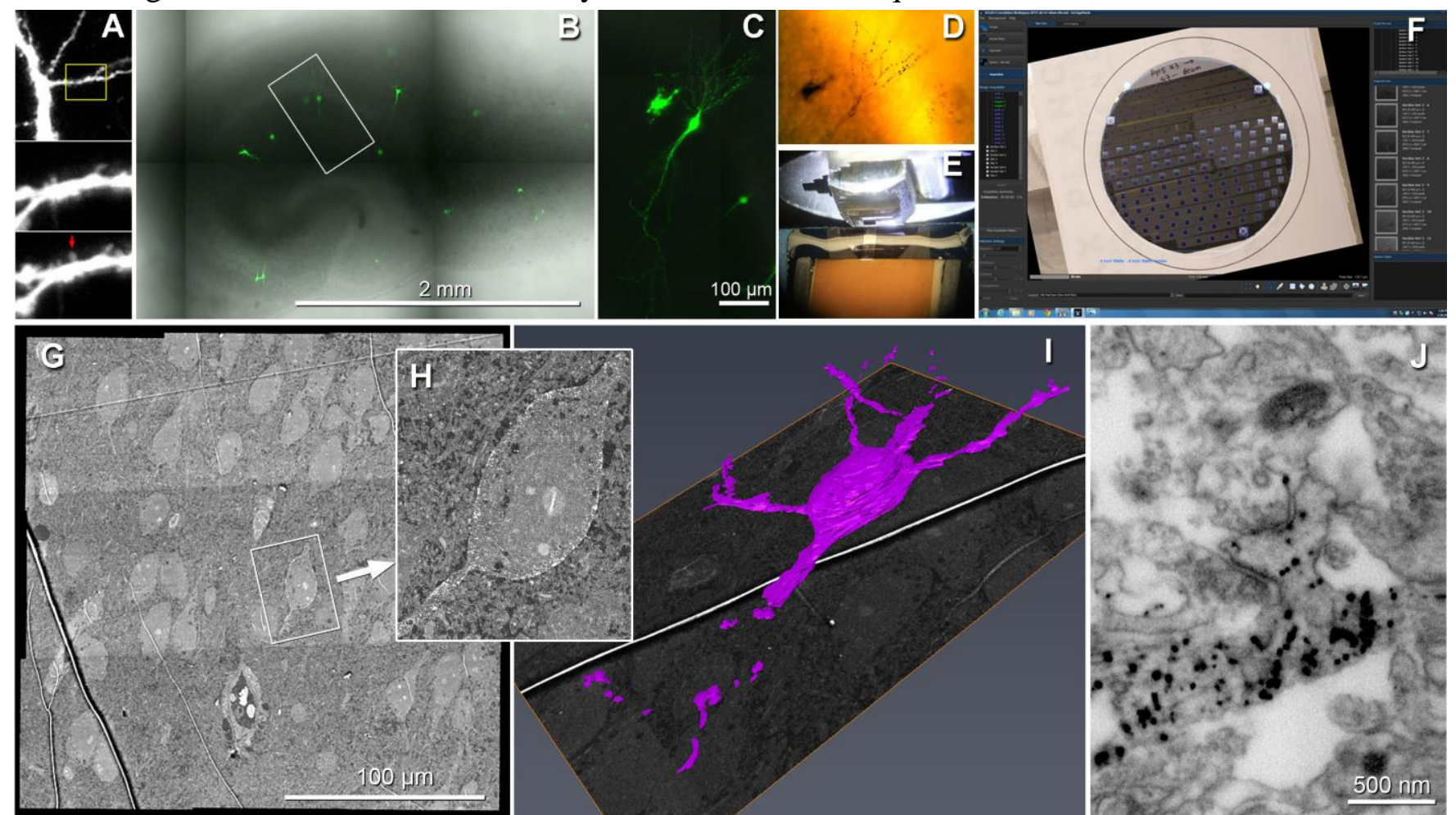

Figure 1. Correlative imaging workflow, A) 2-photon microscope image of a newly synthesized spine (de novo)(arrow) by glutamate uncaging, B) CLSM image of the cultured hippocampal slice containing GFP expressing neurons, C) enlarged image of a pyramidal neuron in the boxed area of (B), D) the same neuron immuno-stained, silver enhanced and embedded in a resin, E) ultrathin sectioning in ATUMtome collecting sections on the tape, F) a screen shot of the Atlas AT5. The first set of low resolution SE2 images were overlaid on the Si wafer image, G) 3x3 tiling BSE images with a $30 \mathrm{~nm} /$ pixel resolution to find target profiles, $H$ ) enlarged image of the boxed area of $(\mathrm{G})$ showing immunogold labeled neuronal soma and a part of its dendrites, I) roughly reconstructed 3D image of the target neuron, J) example of an immunogold-labeled spine taken by the InlensDuo detector with a $5 \mathrm{~nm} /$ pixel resolution.

\section{References:}

[1] KJ Hayworth, JL Morgan, R Schalek, DR Berger, DG Hildebrand and JW Lichtman, Front Neural Circuit. 8 (2014), p. 1.

[2] B Maco, A Holtmaat, A Jorstad, P Fua, and GW Knott in "Correlative light and electron microscopy II", ed. T Muller-Reichert and P Verkade, (Elsevier), p. 339

[3] The authors thank the specialists of Applications and Services from RMC/Boeckeler, Fibics and Carl Zeiss Microscopy for their insensitive supports. 